médecine/sciences $1995 ; 12: 822-4$

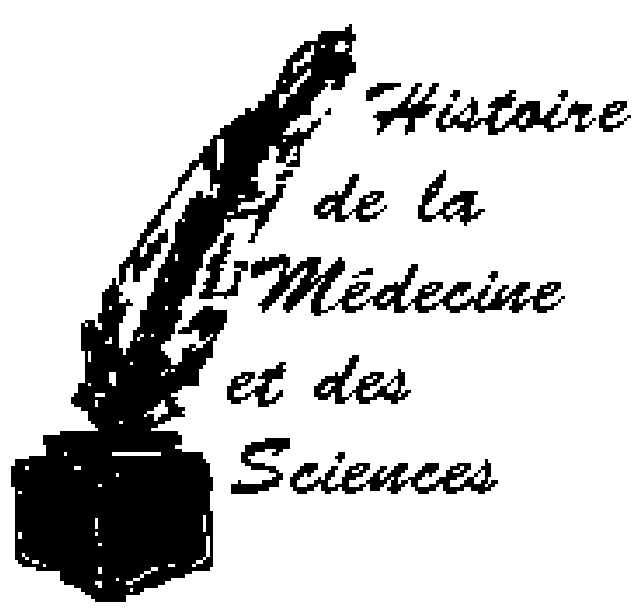

\section{L'invention de la médecine de la douleur}

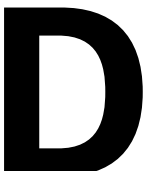

epuis la fin de la Deuxième Guerre mondiale, très lentement, se dessine une nouvelle manière d'appréhender et de traiter la douleur [1]. Des lieux spécialisés dans dans sa prise en charge se développent. En France, les premières "cliniques de la douleur» apparaissent à la fin des années 1970. Dans cet article nous voudrions retracer à grands traits le mouvement qui, entre ces deux moments, conduit à "l'invention d'une médecine de la douleur». Nous suivrons les pas d'un anesthésiste américain, le Docteur John J. Bonica, non qu'il soit le seul acteur de ce mouvement mais parce qu'il en est - au moins s'agissant de la pratique médicale - l'entrepreneur central et, qu'à ce titre, il nous permet de présenter les lignes de force de cette innovation et de son contexte historique (sur l'histoire des recherches sur la douleur et pour la période précédant 1945, on se reportera à Rey [2]). Au cœur du projet de John Bonica se trouve une idée simple: réunir des spécialistes d'origines différentes en une équipe dite "pluridisciplinaire» pour tenter de résoudre des problèmes de douleur complexe pour lesquels les solutions thérapeutiques ordinaires sont restées sans effet (ou, pour le dire plus brutalement, ont échoué). Si l'idée est simple, elle repose cependant sur un changement radical: diriger l'action médicale sur la douleur elle-même et non pas seulement leur l'objet d'un travail médical spécifique, légitime, il fallait la doter de caractéristiques propres qui lui donneraient un nouveau statut. Cela prendra une vingtaine d'années et passera par la séparation d'une douleur clinique de la douleur expérimentale, puis - et c'est essentiel par l'autonomisation de la douleur chronique par rapport à la douleur aiguë. Ce n'est qu'une fois acquise la définition de la douleur chronique comme un "état de maladie» ou une "maladie en soi » (pour reprendre l'expression avancée par Leriche) et établie l'existence de pratiques médicales spécifiques pour y faire face que pourra s'amorcer la mise en place d'un programme de "lutte contre la douleur» sous toutes ses formes, comme on le voit aujourd'hui. Entre temps, la médecine de la douleur sera devenue une réalité.

En 1944, jeune anesthésiste de 27 ans, le Dr John Bonica se trouve du fait d'une guerre, confronté au problème des douleurs rebelles. Constatant l'insuffisance des connaissances disponibles et leur éclatement au sein de la médecine, ainsi que la limite des moyens techniques disponibles (essentiellement les blocs anesthésiques), il forme le projet de constituer une approche clinique spécifique de la douleur pour combler une carence manifeste du système de soins. Dès 1947, revenu à la vie civile, il crée autour du problème de la douleur un groupe informel qui lui sert de laboratoire pour roder l'idée d'une prise en charge pluridisciplinaire de la douleur. Parallèlement il lui faut faire connaître son projet. Il s'appuiera essentiellement sur un livre, The management of pain [3], publié en 1953, dans lequel il cherche à constituer la douleur comme un problème clinique autonome nécessitant que les médecins agissent sur la douleur elle-même, comme on le ferait pour une autre entité morbide.

\section{La douleur: une entité autonome}

Pour convaincre, il doit d'abord dégager la douleur de la double impasse dans laquelle elle se trouve, provenant paradoxalement des progrès conjoints de la science et de la médecine. En effet, les développements simultanés de l'anesthésie, de thérapeutiques efficaces et de nouvelles connaissances scientifiques ont pu laisser penser que le problème de la douleur était à peu près résolu, en même temps qu'ils ont conforté une vision de la douleur comme signal, s'inscrivant dans le paradigme dominant alors la médecine occidentale un symptôme - une cause. De ce fait, l'approche de la douleur se trouve alors circonscrite à la question du diagnostic, et en sont exclus les aspects pénibles, subjectifs. Or, c'est 
justement entre l'expérimental et le subjectif que Bonica cherche à dégager une approche de la douleur sur laquelle puissent travailler les médecins. Il va opposer à la douleur expérimentale une douleur clinique en y intégrant les réactions du malade. Il doit donc dégager la spécificité des conséquences dévastatrices physiques et morales des douleurs rebelles qui interdiront que la douleur soit considérée comme un problème dont on peut se contenter de juger les effets localement. Cependant, il faut le souligner, si la reconnaissance de l'intrication des effets physiques et psychiques de la douleur - reconnaissance qui se manifeste par la nécessité sans cesse réaffirmée d'avoir un psychiatre ou un psychologue dans le groupe - ouvre la voie vers l'autonomisation d'une entité «douleur chronique», elle introduit dès l'origine une zone d'ambiguïté par le double statut qu'elle accorde aux facteurs psychologiques, à la fois élément accompagnateur de toute douleur et facteur causal exclusif de certaines douleurs "psychologiques". De ce double statut naît une ambivalence, voire une ambiguïté, qui sera prise en compte différemment selon les étapes du développement de la médecine de la douleur et selon les acteurs considérés. Pour l'instant, elle constitue un outil pour Bonica pour faire tenir un objet douleur qui, loin du laboratoire, peut intégrer l'individu et son expérience vécue et ouvrir, au prix d'un renoncement à certaines habitudes de pensée, un espace nouveau au travail médical le plus légitime, c'est-à-dire s'appuyant sur les données scientifiques les plus récentes.

\section{Une médecine \\ de la «personne totale»}

Malgré d'inlassables efforts qui le transforment en véritable «commis voyageur de la douleur», Bonica rencontre d'abord peu d'échos. Ce n'est que dans la fin des années 1960 et au début des années 1970 que la situation bascule. Certains facteurs aident à saisir ce renversement. En premier lieu, l'organisation de la médecine: si les développements de l'anesthésie permettaient de proposer des tech- niques, celui plus large de la spécialisation [4], se faisant dans un découpage par organe ou grand appareil, a dans les années 1950 pesé contre le projet de Bonica. La douleur, en effet, comme importante donnée sémiologique, suivait le même découpage qui la rendait littéralement non visible en tant qu'entité propre. Avec le temps, cependant, ce phénomène d'hyperspécialisation provoquera les conditions d'une visibilité de la douleur qui dure comme problème de santé qui n'est plus pris en charge par personne. Par ailleurs, Bonica, en partant de la douleur vécue, rejoint un autre courant de transformation qui s'opère autour de la place de l'individu dans la pratique médicale. De nombreuses recherches historiques $[5,6]$ mettent en lumière la manière dont l'approche clinique et la médecine dite "moderne» se sont construites en évinçant le malade comme sujet de leur champ d'investigation. Cependant, selon certains auteurs [5-7], un mouvement inverse, prenant la forme d'un «retour de la personne sentant», s'amorce depuis le début des années 1950, comme en témoigne la montée d'une nouvelle logique médicale qui intègre ce qui était à l'extérieur du regard médical («l'individu anormal » dont la place n'était pas à l'hôpital) et le transforme en un malade dont le problème total devient le souci de la médecine. Cette transformation s'opère au travers de la fusion de deux discours normatifs sur la vie, jusque-là distincts tout au long du XIX ${ }^{\mathrm{e}}$ siècle, le discours médical et le discours sociomoral de la compassion, fusion qui, en remplaçant dans la pratique médicale la catégorie de l' «anormal» par celle du «malade chronique", fait de l'expérience subjective de la maladie une partie intégrante de la médecine et fait de «la personne totale» un objet de l'attention médicale. Cette nouvelle logique redéfinit le malade, le médecin et la maladie et appelle une réorganisation de la pratique médicale qui prend forme autour de l'idée d'une gestion de la maladie faite par une «équipe de santé » dont l'approche est moins morcelante. La première formulation des pain clinics s'inscrit déjà dans ce mouvement.

\section{Neurophysiologie de la douleur: la gate control theory}

C'est dans ce contexte historique large que vont converger certains facteurs pour faire concrètement décoller le projet de Bonica. Le plus important est, en 1965, la publication d'une nouvelle théorie de la douleur, la théorie de la porte ou gate control theory, par Melzack et Wall [8]: «Fondamentalement, cette théorie propose l'existence d'un mécanisme agissant comme une barrière dans le système de transmission synaptique de telle sorte que les signaux de douleur puissent être modulés avant qu'ils ne déclenchent une perception et une réponse. La barrière peut être ouverte ou fermée à des degrés divers en fonction de facteurs comme l'activité relative des fibres périphériques, grosses et petites, et des processus psychologiques variés comme l'attention ou l'expérience antérieure. En proposant une barrière modulable, il devenait possible d'essayer de fermer la barrière par des manipulations diverses », changeant ainsi profondément la représentation de la douleur et ouvrant des voies thérapeutiques nouvelles. Parallèlement, et c'est un point sociologique important, la reconnaissance du poids médical, social et économique des maladies chroniques attire l'attention sur la nécessité d'imaginer des formes nouvelles de gestion, de contrôle de ces maladies. Dans ce cadre, la naissance des unités de soins palliatifs (en 1967, création du Saint Christopher Hospice) organisées autour de la notion d'équipe fait écho dans un autre champ à l'approche pluridisciplinaire de Bonica et sensibilise au problème de la prise en charge de la douleur. Par ailleurs, un événement fortuit, le voyage du président Nixon en Chine en 1972, à travers la question des phénomènes d'anesthésie par acupuncture, braque les projecteurs sur la question de la douleur entraînant la première attribution spécifique de crédits venant des National Institutes of Health. Enfin, les résultats d'importantes recherches (dont en 1973 la découverte des récepteurs opiacés, après 1975 , de leurs ligands endogènes) [10] viennent à point pour soutenir le travail de Bonica, tant par l'excitation suscitée dans 
le milieu de la recherche que par les applications cliniques qui paraissaient très prometteuses. Toute cette effervescence profite au travail de rassemblement entrepris par Bonica en attirant l'attention des chercheurs sur la douleur et, associée à l'intérêt suscité par la théorie du gate-control, elle va lui permettre de sensibiliser quelques cliniciens. En mai 1973, il réunit à Seattle-Issaquah dans l'État de Washington (USA) 350 personnes d'origines disciplinaires variées, exclusivement autour de la douleur. C'est un moment essentiel dans l'histoire de la médecine de la douleur: on assiste à un changement de régime d'une nébuleuse d'intérêts individuels pour la douleur à l'installation d'un monde, un milieu spécifique qui prendra la forme d'une association scientifique, véritable «internationale de la douleur».

\section{La douleur chronique et sa double origine, physique et psychogène}

A partir de là l'histoire est mieux connue: c'est la création, un an plus tard, de l'IASP (Association internationale pour l'étude de la douleur), puis la naissance de la revue Pain, et à la fin des années 1970 l'émergence, en France, des premières consultations de la douleur. La médecine de la douleur est née. Avant de conclure, il faut s'arrêter sur un point encore: c'est au cours de cette même réunion que la douleur chronique en tant qu'entité propre, distincte de la douleur aiguë, a été vraiment reconnue grâce à l'aide de certains psychiatres et surtout de psychologues cliniciens, dont Fordyce et Sternbach, permettant de bien marquer le premier territoire de la médecine de la douleur. Mais, en même temps qu'ils donnaient des arguments pour reconnaître les spécificités de la douleur chronique en définissant la douleur comme un comportement, ces psychologues proposaient pour lire la douleur un autre modèle théorique que celui de la biomédecine, introduisant une tension conceptuelle dans ce champ. Sur le plan théorique, la médecine de la douleur, depuis 1973, aborde la douleur chronique à partir de deux modèles. Le premier est médical stricto sensu; il part de la douleur, manifestation physique incluant des éléments individuels liés à la personnalité du malade ou à ses circonstances de vie influençant le vécu de sa douleur, et il admet pour certaines douleurs - les douleurs psychogènes - une origine non physique. L'autre modèle est comportemental: il part de l'individu et de son comportement comme opérateur de toute douleur chronique quelle qu'en soit l'origine, physique ou non et, si l'origine est physique (une maladie ou une lésion), que cette cause soit éteinte ou non. Autrement dit, il y aurait deux modes d'entrée dans la douleur, un par le corps, l'autre par la personne et sa douleur qui soustendent les pratiques des différents centres de la douleur. C'est dans ce sens qu'on peut parler d'une introuvable définition unifiée de la douleur chronique et d'une tension qui traverse, jusqu'à aujourd'hui, la médecine de la douleur

\section{RÉFÉRENCES}

1. Baszanger I. Douleur et médecine, la fin d'un oubli. Paris : Seuil, 1995.

2. Rey R. Histoire de la douleur. Paris: La découverte, 1994.

3. Bonica JJ. The management of pain. Philadelphia, London: Lea and Febiger, 1953.

4. Reiser SJ. Medicine and the reign of technology. Cambridge: Cambridge University Press, 1978.

5. Armstrong D. The patient's view. Soc Sci Med 1984; 18: 737-44.

6. Jewson ND. The disappearence of the sick man from medical cosmolgy 1770-1870. Sociology 1974 ; 10 : 225-44.

7. Arney WR, Bergen BJ. The anomaly, the chronic patient and the play of medical power. Soc Health Illness 1983; 5 : 1-24.

8. Arney WR, Bergen BJ. Medicine and the managment of the living. Taming the last great beast. Chicago: Chicago University Press, 1984.

9. Melzack R, Wall PD. Pain mechanisms. A new theory. Science $1965 ; 150$ : 971-9.

10. Besson JM. La douleur. Paris: Odile Jacob, 1992.

\section{Isabelle Baszanger}

Centre de Recherche Médecine Maladie et Sciences Sociales, Cnrs UM 043, EHESS division sociologie, Inserm $U$. 304, 182, boulevard de la Villette 75019 Paris, France.

\section{TIRÉS À PART}

I. Baszanger. 